\title{
Supporting Exploratory Learning with Questioning and Computational Thinking in Integrated STEM Education
}

\author{
Qian Qian, Yuan Liu*, Hao Kun \\ School of Computer Science, Shaanxi Normal University, Xi'an, China \\ Email address: \\ Qianqianer369@163.com (Qian Qian), yuanliu@snnu.edu.cn (Yuan Liu),kkawake@163.com (Hao Kun) \\ ${ }^{*}$ Corresponding author
}

To cite this article:

Qian Qian, Yuan Liu, Hao Kun. Supporting Exploratory Learning with Questioning and Computational Thinking in Integrated STEM

Education. International Journal of Elementary Education. Vol. 10, No. 3, 2021, pp. 93-99. doi: 10.11648/j.ijeedu.20211003.17

Received: July 22, 2021; Accepted: September 10, 2021; Published: September 15, 2021

\begin{abstract}
Exploratory learning is a type of active learning by supporting learners in freely exploring relevant instructional resources; learners take a more active role in their learning rather than being passive receivers of information. It's a popular pedagogical method for integrated STEM education that involves multiple disciplines and knowledge domains. However, empirical evaluations have shown that exploratory learning is not always effective for all learners and that some learners may benefit from more structured, explicit instructions as more traditional intelligent tutoring support. In this paper, we focus on how to support the effective knowledge dissemination and learning results of learners in the process of autonomous exploratory learning without weakening the role of learners in an active learning environment. A practical strategy to support exploratory learning in integrated STEM education was proposed by combining scaffolding questioning and Computational Thinking (CT). First, we analyze how to optimize teachers' questioning strategies to spread knowledge effectively and make learners' learning process more active. Then, how to take advantage of the benefits of CT was addressed. Finally, we construct a Problem-Oriented Learning Model of Integrating CT (POLMICT) suitable for K-12 STEM education. By applying POLMICT to STEM course in middle school, we concluded that scaffolding questioning strategies can be used as a bridge for teachers to impart knowledge to learners to improve the effectiveness of exploratory learning, and embedding $\mathrm{CT}$ in this process helps learners to explore and ensure their positive role in the STEM environment.
\end{abstract}

Keywords: STEM Education, Exploratory Learning, Computational Thinking, Scaffolding Questioning, Learning Process

\section{Introduction}

STEM is the abbreviation of Science, Technology, Engineering and Mathematics. STEM education helps students master knowledge and skills in an integrated teaching method, and promotes students' flexible migration to solve practical problems. STEM education emphasizes interdisciplinary integration. Its core characteristics are interdisciplinary, interesting, experiential, contextual, collaborative, design, and empirical [1]. In integrated STEM education, exploratory learning is a type of active learning involving multiple disciplines and knowledge domains. Active learning is manifested as: under the drive of individual need, learners freely explore relevant instructional resources, persistently carry out self-learning, self-assessment, and self-monitoring, thereby improving learning efficiency [2]. It is conducive to the development of learners' Computational Thinking (CT), the formation of problem-solving ability, and the establishment of life-long learning concepts. Moreover, STEM education and learners' exploratory learning pursues the same goal. STEM education is full of the concept and spirit of exploratory learning [3], which can support practical activities of exploratory learning.

The existing STEM education models and methods are diverse. Although they promote students' interest in exploration to a certain extent, they do not ensure the effectiveness of students' exploratory learning results. Moreover, the empirical evaluation shows that autonomous exploratory learning is not always effective for all learners, but some learners may benefit from more structured and clear instructions. In other words, effective exploratory learning not only requires teachers to give students more inquiry space and stimulate their inquiry interest, but also requires teachers to provide appropriate guidance and promote 
students' development of high-order thinking. Based on the above analysis, we find that: in the K-12 stage of STEM education, how to ensure the effectiveness of knowledge dissemination and learning effectiveness of learners in the process of independent exploratory learning without weakening the learners' enthusiasm for active inquiry in the learning environment is an important problem. Therefore, based on the constructivist learning theory, this paper constructs a Problem-Oriented Learning Model of Integrating Computational Thinking (POLMICT) suitable for K-12 STEM education, which combines scaffolding questioning with CT to support exploratory learning, ensure learners' autonomy in exploratory learning, and improve the effectiveness of learners' learning outcomes.

\section{Related Work}

The second part of this article mainly adopts the literature research method to define and explain the research status of several concepts of STEM education, Exploratory learning, Questioning, and CT, which leads to the purpose of this article.

There are three main learning methods in integrated STEM education: Problem-based Learning (PBL), Project-based Learning (PJBL), and Hand-on Inquiry-Based Learning (HIBL) [1]. The above three learning methods all require students to maintain the enthusiasm of independent exploration in the learning process to cultivate students' CT and solve real problems of interdisciplinary in real life.

Exploratory learning is an effective path to carry out STEM education and is characterized by "independence, inquiry, and cooperation" [3]. Zhao H C [4] and others believe that STEM education should be full of the concept and spirit of exploratory learning and support practical activities of exploratory learning. For this reason, Catarina Correia et al. [5] emphasized that teachers need to arrange the content of inquiry reasonably, highlight the method of inquiry, and emphasize the penetration of inquiry thinking to achieve the goal of exploratory learning. M Chen et al. [6] believe that STEM education can support students to master knowledge and skills in the process of solving real problems through the integration of disciplines and to conduct creative exploration. At present, the concept of exploratory learning has shifted from confirmatory inquiry to creative inquiry [4]. However, the practice of exploratory learning in my country's compulsory education stage still has a phenomenon of anomie [4], which is manifested as insufficient inquiry spirit and low inquiry efficiency. Therefore, we need to face up to the problems existing in primary and secondary school students' exploratory learning, that is, autonomous exploratory learning is not always effective for all learners. For example, learners who are field-dependent or have a low sense of self-efficacy rely more on the teacher's leadership in their learning and prefer teachers to impart their knowledge. One of the research purposes of this article is to find effective solutions to such problems.

The key to PBL lies in problem design, and "problem driving" focuses on constructing a "problem chain" [7]. Tom Bielik T et al. [8] believe that in STEM education, exploratory learning requires teachers to create situations and stimulate students' desire to explore, and questioning is one of the important tools for teachers. Rim Kyung hwa et al. [9] proposed that strategic questioning refers to the selection and application of questions with a clear effect. In STEM education, problem-based teaching is problem-driven and learner-centered teaching [10]. Of course, different questioning strategies will also have different effects on students' exploratory learning. To achieve the goal of exploring, teachers need to use strategies with questions. This article aims to develop a questioning strategy, which helps teachers improve their understanding of various questioning processes and thinking abilities, and helps teachers promote students' reflection and the development of CT skills through scaffolding questions.

Jeannette Wing [11] first proposed the concept of CT and defined CT as a mode of thinking. Joseph Lyon et al. [12] believe that CT is not only applicable to the concepts and thinking of computer science, but also provides a perspective that is widely used to analyze problems in work, study, and life. CT includes five elements: decomposition ability, generalization ability, algorithm thinking, evaluating ability, and abstraction [13]. The teaching process of STEM education is divided into five steps: identify problems, planning, trying, modifying, and communicating [14]. Zhu K et al. [15] believe that the teaching process of STEM education needs the support of CT ability, and at the same time, exploratory learning will also cultivate students' CT ability. At present, domestic research on the cultivation of CT begins at the general higher education, but in the K-12 stage, the application and cultivation of CT ability in the teaching process are still in the initial stage. This article constructs POLMICT suitable for the K-12 stage, which helps teachers use questioning strategies and CT to organize teaching; promotes students to use thinking methods to construct knowledge and skills and cultivate CT capabilities.

\section{Method}

A practical method to support exploratory learning in integrated STEM education was proposed by constructing a Problem-Oriented Learning Model of Integrating Computational Thinking (POLMICT) suitable for K-12 STEM education, which combines scaffolding questioning with $\mathrm{CT}$.

\subsection{Scaffolding Questioning Strategies in STEM Education}

In STEM education, PBL, PJBL, and HIBL start from a problem that needs to be solved [7]. This problem is called a Driving Question (DQ). Students explore the DQ and learn and apply subject ideas in the process of exploring. There are multiple paths to achieve the goal of PBL, PJBL, and HIBL, and sometimes even goals are relatively vague. The cognitive level of elementary and middle school students is limited. Therefore, teachers need to use scaffolding questions to detail and optimize these problems, projects, and designs of the STEM course. When the questioning path is determined and unique, students are prone to a sense of powerlessness and 
dependence, and the motivation to creatively solve problems is attenuated; when the questioning path is uncertain or diversified, it is easy to stimulate students' motivation and make them fully integrated into innovation during the problem-solving experience.

The characteristic of effective scaffolding questions is that the goal is clear, while the plan is vague [16]. It also provides a learning scaffold for students to explore independently. Specifically:

Teachers provide students with interesting learning situations that enable students to generate, think, and solve problems.

Teachers design scaffolding questions or related "question chains" according to the teaching content to guide and promote students' thinking activities.

Teachers provide students with clues about problems through scaffolding questions, stimulate students' interest, guide students to explore and learn independently, and find solutions to problems.

Teachers guide students to develop a deeper understanding of asking questions and be able to summarize and evaluate their problem-solving methods.

Teachers evaluate the performance of students in the process of problem exploration and evaluate the effect of students' exploratory learning.

\subsection{CT in STEM Education}

The convergence of CT and STEM education lies in interdisciplinary and problem-solving [13]. Cultivating students' problem-solving ability is one of the important educational goals of the K-12 education stage. CT, as a way of problem-solving thinking, is the ability that all educated persons should possess [12]. The thinking of elementary and middle school students is constantly developing. The cultivation of their CT needs to be combined with the concept of interdisciplinary knowledge integration of STEM education, starting from all aspects, and organically integrating with multiple disciplines. At the same time, the teaching process of STEM education also needs the support of CT ability. The basic CT ability of students also promotes the completion of learning tasks and the achievement of learning goals.

When cultivating primary and middle school students' CT, teachers need to purposefully introduce questions or create situations to stimulate students' active inquiry and cultivate students' ability to solve interdisciplinary problems. To better implement STEM education, it is necessary to cultivate students' CT ability in the teaching process of STEM education. Meanwhile, the key steps in the STEM teaching process also need the support of CT elements. CT includes five elements: decomposition ability, generalization ability, algorithm thinking, evaluating ability, and abstraction [13]. The teaching process of STEM education is divided into five steps: identify problems, planning, trying, modifying, and communicating [14]. The relationship between CT and STEM Education is shown in Figure 1.

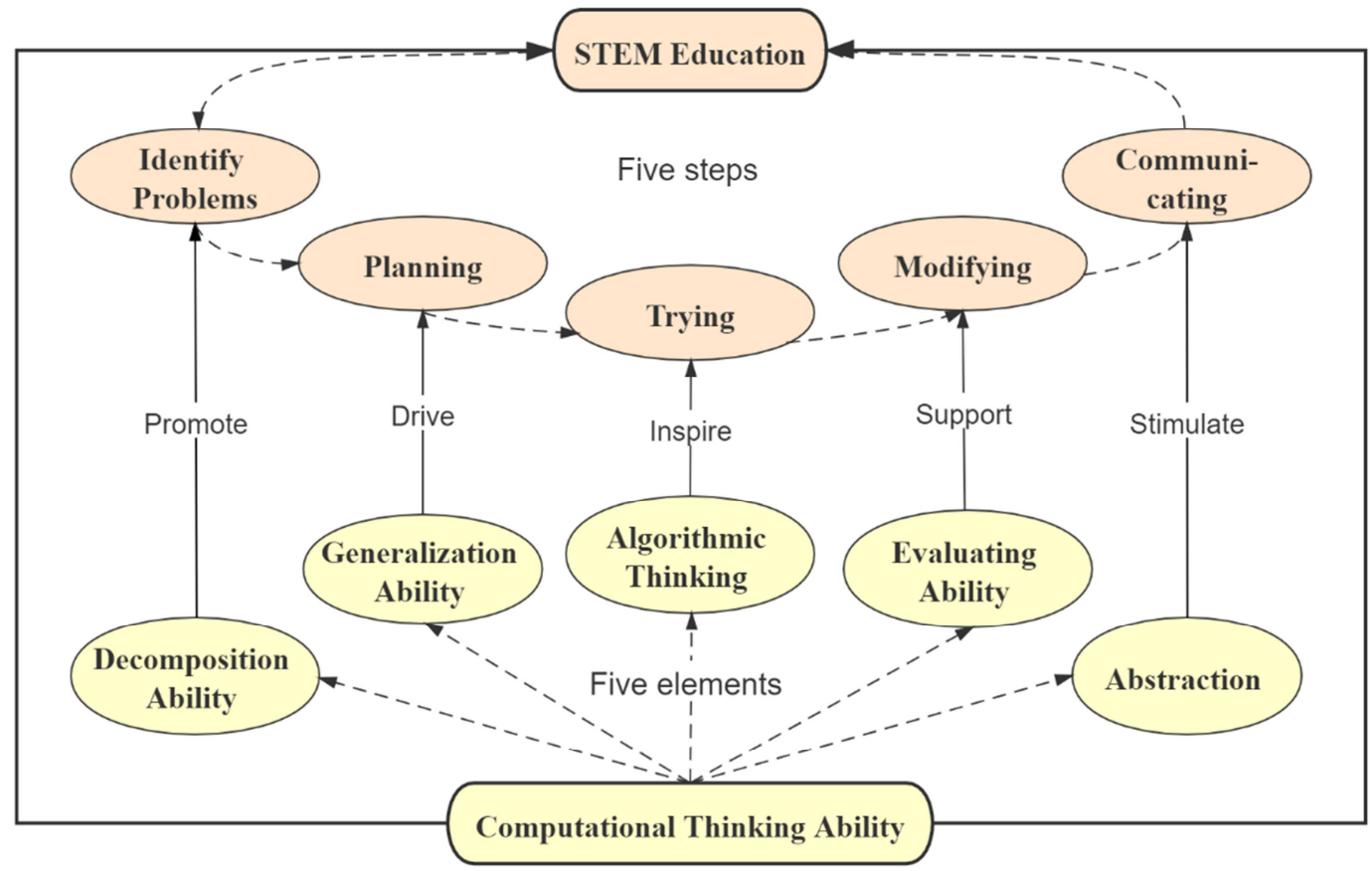

Figure 1. The Relationship between CT and STEM Education.

\subsection{Scaffolding Questioning \& CT in STEM Education}

Incorporating the concept of $\mathrm{CT}$ into the teaching or learning process of STEM education [15], teachers construct learning scaffolds for students through effective questioning, and students use CT methods to conduct inquisitive learning to better exert the effectiveness of both. Comprehensive use of CT teaching strategies to construct a new teaching model with teachers as the leading, student as the main body, and problem-solving ability training as the goal----The Problem-Oriented Learning Model of Integrating 
Computational Thinking (POLMICT).

The POLMICT uses CT as the entry point, is led by exploratory learning, and takes "question" as the mainline to connect the process of teacher teaching and student learning. The POLMICT requires teachers to use CT methods to ask questions to guide students in the process of exploratory learning and provide effective resources to help students explore their learning tasks. Students should use CT methods to sort out their knowledge structure, explore learning actively, complete learning tasks, and construct their learning mode. By the POLMICT, students can better carry out independent, collaborative and exploratory learning, master knowledge, and achieve the goals of knowledge transfer and thinking ability training.

The POLMICT is mainly constructed by "One Scaffold", "One Core", "Two Main Bodies" and "Five Stages" as shown in Figure 2.

One Scaffold: The teacher's questioning is used as a scaffold for students' exploratory learning. The design points are: (1) consistent with the students' original learning experience related to $\mathrm{CT}$, and the scaffold provided should be placed in the student's "recent development zone"; (2) reflect the teachers' CT process and provide feedback for students to answer the question and solve the problem.

One Core: Teachers use CT to organize teaching, and students use a series of CT methods to conduct self-inquiry and study to achieve the goal of interdisciplinary problem-solving.

Two Main Bodies: Teachers are question designers, problem exploration organizers, and problem-solving guides; students are active subjects who use CT to think, analyze, and solve problems.

Five Stages: Teaching activities based on POLMICT can be divided into five stages: preparations, questions, analyses, solutions, and summaries. The corresponding CT capabilities of each stage are decomposition ability, generalization ability, algorithmic thinking, evaluating ability, and abstraction.

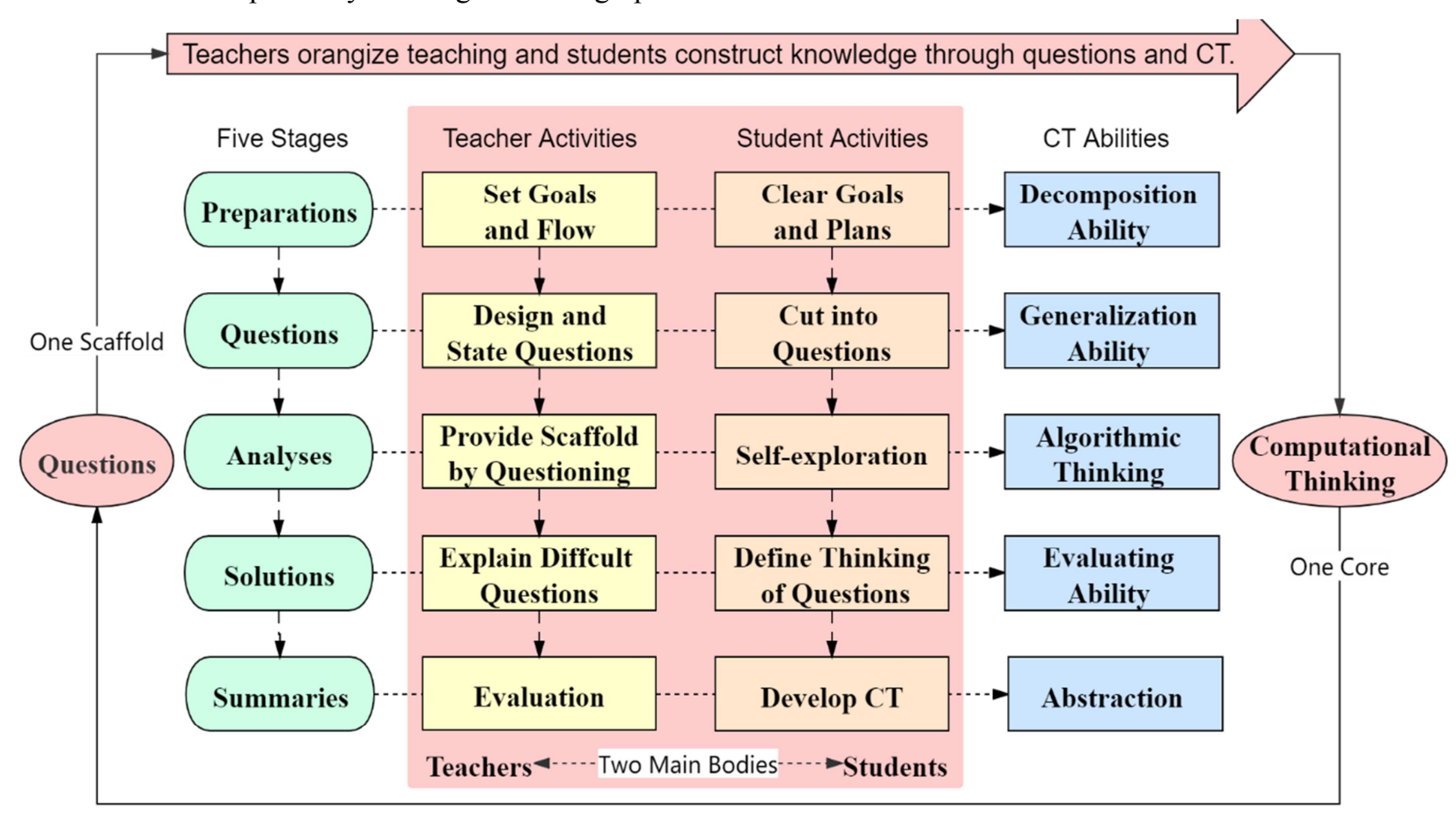

Figure 2. The Problem-Oriented Learning Model of Integrating CT (POLMICT).

\section{Application}

The fourth part mainly adopts the comparative experiment method, we use POLMICT and General Inquiry Teaching Model (GITM) to design and implement an example of the junior high school STEM course "Protecting Eggs----Redundancy and Fault Tolerance in STEM Exploration" respectively for the classes with the same initial situation of students. This course covers the fields of Science, Technology, Engineering, Art, Biology, and Physics. Students review various phenomena of falling from a height in their lives, think about the damage caused by falling objects, and think about ways to solve such problems in real life.

\subsection{Exploratory Learning Activity Process Based on POLMICT}

According to POLMICT, in a class, teachers use scaffolding questions to guide students to explore independently and use CT methods to design buffer methods and structures to protect eggs, to solve the interdisciplinary problem of how to protect objects falling from height in the real situation. The exploratory learning activity process of "Protecting Eggs" based on POLMICT and the corresponding CT abilities are shown in Table 1.

In the Analyses stage, the inquiry task, materials, and scaffolding questions presented by the teacher to the students 
are shown in Table 2.

Table 1. "Protecting Eggs" Exploratory Learning Activity Process Based on POLMICT.

\begin{tabular}{|c|c|c|c|}
\hline Teaching stage & Teacher activities & Student activities & CT abilities \\
\hline Preparations & Set goals and flow & Clear goals and plans & Decomposition ability \\
\hline Questions & Design and state questions & Cut into questions & Generalization ability \\
\hline Analyses & Provide scaffold by questioning & Self-exploration & Algorithmic thinking \\
\hline Solutions & Explain difficult questions & Define thinking of questions & Evaluating ability \\
\hline Summaries & Summary and evaluation & Migration and internalization & Abstraction \\
\hline
\end{tabular}

Table 2. Introduction to the "Protecting Eggs" Exploratory Learning Activity Based on POLMICT.

\begin{tabular}{|c|c|}
\hline Project & Content \\
\hline Inquiry task & Make a ground buffer device as a team so that the eggs will not break when falling from a height of 1.5 meters. \\
\hline Materials & $\begin{array}{l}\text { Raw egg, string, straw, newspaper, plasticine, plastic wrap, chopsticks, plastic bag, rubber band, bubble film, tape, scissors, } \\
\text { cotton cloth }\end{array}$ \\
\hline Scaffolding questions & $\begin{array}{l}\text { Is the cushioning device softer or harder better? What level of a buffer do you think is the most appropriate? } \\
\text { What will happen if the cushioning device is made into a "trampoline"? } \\
\text { What will happen if the buffer device is convex in the middle and concave around it? } \\
\text { In what ways did the eggs hit the ground? } \\
\text { Which buffer devices can be used on eggs? } \\
\text { What is the fault tolerance rate of your structure? } \\
\text { Can you design a buffer device that is easy to move and quickly lay to reduce the damage caused by falling objects? }\end{array}$ \\
\hline Explore time & Design and production (20 minutes), revised design (10 minutes) \\
\hline Evaluation index & $\begin{array}{l}\text { Completion time, small group work and cooperation, the feasibility of the design plan, the situation after the egg has landed, } \\
\text { the production process and aesthetics of the device, and the summary display effect }\end{array}$ \\
\hline Achievement display & $\begin{array}{l}\text { Show the best-performing buffer device, draw both successful and failed designs on the workbook, and reflect on the failed } \\
\text { model. }\end{array}$ \\
\hline
\end{tabular}

\subsection{Exploratory Learning Activity Process Based on GITM}

On the contrary, we adopt the GITM, set the same class hours, and design and implement this lesson example. The exploratory learning activity process based on GITM is shown in Table 3.

Table 3. "Protecting Eggs" Exploratory Learning Activity Process Based on GITM.

\begin{tabular}{lll}
\hline Teaching stage & Teacher activities & Student activities \\
\hline Create a situation & Stimulating engine & Enter the situation \\
Inspire thinking & Provide guidance & Form a plan \\
Self-inquiry & Monitor the inquiry process & Processing information \\
Communication & Organize discussion & Discuss, share \\
Summary & Summary and evaluation & Summary comments \\
\hline
\end{tabular}

\subsection{Comparative Analysis of GITM and POLMICT}

The above two models correspond to five teaching stages. According to the evaluation index in Table 2, the teacher evaluated the performance of exploring and the results displayed by the students in each class. The results show that each stage of GITM and POLMICT has significantly different effects on the effect of students' exploratory learning. Specifically:

The first stage: GITM corresponds to "Create a situation". Teachers and students interact only through a situation. Students have not yet formed the psychological preparation for exploratory learning, and the ability required at the beginning of exploring has not been stimulated. While "Preparations" corresponding to POLMICT require teachers to introduce the DQ to inspire students to think actively and stimulate students' interest. Students use CT methods to clarify and decompose the DQ of exploratory learning: "How to design a buffering method and structure for falling objects from high altitude to ensure that the falling objects are in good condition", and formulate the next step of the exploration plan. The CT ability corresponding to this stage is the decomposition ability.

The second stage: GITM corresponds to "Inspire thinking". Since there is no clear tool and method for teachers to inspire students to think, the depth and breadth of students' independent thinking are limited. While "Questions" corresponding to POLMICT require teachers to design and restate the DQ and throws out scaffolding questions or "question chains". E.g. (1) What kind of damage does falling from a high altitude cause to falling objects? (2) What happens when an eagle, a rabbit, and an ant fall from a height? (3) What are the ways to slow downfalls in life? The above problems provide students with a certain degree of exploratory learning support. Students use CT methods to find the entry point to solve the DQ and construct a method to protect falling objects from high altitudes in their minds. The CT ability corresponding to this stage is the generalization ability.

The third stage: GITM corresponds to "Self-inquiry". Since there is no clear content and form for teachers to guide 
students' autonomous inquiry, the enthusiasm and effectiveness of students' autonomous inquiry are not guaranteed. While "Analyses" corresponding to POLMICT require teachers to present materials such as inquiry tasks and scaffolding questions, as shown in Table 2. Students organize and analyze their plans according to the relevant resources provided by the teacher, and conduct self-exploration. The $\mathrm{CT}$ ability corresponding to this stage is algorithmic thinking.

The fourth stage: GITM corresponds to "Communication". Teachers simply organize discussions and students share mechanically, which does not give full play to the interaction between teachers and students. While "Solutions" corresponding to POLMICT require each group of students summarized some of the problems (or unsuccessful points) found in the process of independent exploration, which may include the following: (1) The egg fell from the buffer and was damaged. (2) The cushioning device is very soft, but the egg is still damaged. (3) The egg did not fall in the center of the buffer device. On this basis, teachers use CT methods to explain these difficult problems to students in a targeted manner. Under the help and guidance of teachers, students test and revise their designs, perfect the design scheme of the egg protection device, and clarify the idea of solving inquiry questions. The CT ability corresponding to this stage is the evaluation ability.

The fifth stage: GITM corresponds to "Summary". Teachers fail to use questioning strategies effectively, and the effectiveness of students' knowledge and skill construction cannot be accurately assessed. While "Summaries" corresponding to POLMICT requires students to apply newly learned scientific concepts to communicate. E.g. change the shape of the buffer device to reduce the impact force received when the egg falls, or increase the redundant design and fault-tolerant design of the buffer structure to ensure the effect. After presenting, students can describe enough details to make their methods easier to understand by others, and can clearly explain what factors affect the performance of the cushioning device. In addition to the engineering design of the device itself, teachers also need to pay attention to the written report materials formed by the students in the inquiry activities; what students experience is the scientific inquiry, interdisciplinary problem solving, and development of the process of CT. The CT capability corresponding to this stage is Abstraction.

\section{Conclusion}

The above application shows that students can also complete the given exploratory task within the specified time under GITM. However, due to the lack of learning scaffold and CT, students' initiative and creativity have not been fully utilized, and interdisciplinary problem-solving ability has not been fully developed. While in POLMICT, students start from the cognitive conflicts caused by realistic DQ. By experiencing the complete process of an inquiry task from beginning to end, it is easy to understand the factors that affect the performance of the cushioning device and to solve similar interdisciplinary problems through migration and internalization. While solving teaching problems, it also cultivates students' interdisciplinary problem-solving ability and CT ability. The practice of POLMIC has proved that the use of scaffolding questioning strategies in exploratory learning can not only ensure the effectiveness of learners' construction of knowledge and skills but also can promote learners' development CT. At the same time, in the process of exploratory learning of different learners, learners who have basic CT have shown great advantages in terms of linking different disciplines, designing problem solutions, and integrating resources. All this preliminarily confirms the argument that "scaffolding questioning strategies can be used as a bridge for teachers to impart knowledge to learners to improve the effectiveness of exploratory learning, and embedding CT in this process helps learners to explore and ensure their positive role in the STEM environment". Therefore, POLMIC can be used as an effective model to support students' exploratory learning by combining scaffolding questioning and CT in the K-12 education stage.

Although the construction and application cycle of POLMIC is limited, it can be optimized through further research. E.g. we can focus on (1) How to optimize teachers' scaffolding questioning strategies, how to formulate, display and evaluate scaffolding questions? (2) How to promote teachers to use CT to organize teaching and students to use CT to carry out exploratory learning? (3) How to accurately evaluate the effectiveness of learners' use of POLMICT for exploratory learning. Furthermore, the concepts of STEM education, exploratory learning, and CT are introduced into practical teaching to give full play to its teaching effect and provide a reference for its practical application.

\section{Acknowledgements}

This study was supported by research grants funded by the National Natural Science Foundation of China (Grant No. 61907029) and Natural Science Foundation of Shaanxi, China (Grant No. 2020JM-307).

\section{References}

[1] Holmlund T D, Lesseig K, Slavit D. Making sense of STEM education in K-12 contexts [J]. International Journal of STEM Education, 2018, 5 (1)

[2] Keeley-Webb Copridge, Uttamchandani Suraj, Birdwell Tracey Faculty Reflections of Pedagogical Transformation in Active Learning Classrooms [J]. Innovative Higher Education, 2021, 46 (2): 205-221.

[3] J Qin, Gangshan FU, Education S O, et al. Research on Design-based Learning for STEM Education: Model Construction and Case Analysis [J]. e-Education Research, 2018.

[4] Zhao H C. The Design and Implement of Middle School Students' Inquiry Learning from the Perspective of STEM Education [J]. Modern Educational Technology, 2017. 
[5] Catarina Correia, Harrison Christine. Teachers' beliefs about Inquiry-based learning and its impact on formative assessment practice $[\mathrm{J}]$. Research in Science and Technological Education, $2019,38$.

[6] M Chen, Chen Z, School S-Y. A-STEM: A Value Reconstruction of the Interdisciplinary Integrative Education Mode [J]. Research in Educational Development, 2019 (06), $15-22$

[7] Shannon Navy, Kaya Fatma. PBL as a pedagogical approach for integrated STEM: Evidence from prospective teachers [J]. School Science and Mathematics, 2020, 120221-232.

[8] Bielik T, Yarden A. Promoting the asking of research questions in a high-school biotechnology inquiry-oriented program $[\mathrm{J}]$. International Journal of STEM Education, 2016, 3 (1): 15.

[9] Rim Kyung hwa, An Jung hyun. Design of Flipped Learning with Strategic Questioning to Improve Student's Problem-Solving Competency in Engineering [J]. Journal of Practical Engineering Education, 2016, 8 (2).

[10] Zhong Qi, Tang Yao, Zeng Xiangzhi. The Teaching Strategies and Practice Exploration of STEM Based on PBL [J]. Digital Education, 2020, (04), 78-83.
[11] Jeannette Wing. Computational Thinking [J]. Communications of the ACM, 2006, 4933-35.

[12] Joseph Lyon, Magana Alejandra. Computational thinking in higher education: A review of the literature [J]. Computer Applications in Engineering Education, 2020, 28.

[13] Michael Vallance, Towndrow Phillip. Computational thinking and interdisciplinary learning: time to focus on pedagogy [J]. International Journal of Learning Technology, 2020, 15180-200.

[14] F Fernandez, Arco-Tirado Jose, Carrillo-Rosua Javier, et al. Making STEM Education Objectives Sustainable through a Tutoring Program [J]. Sustainability, 2020, 126653.

[15] Zhu K, Jia X X, University H N. Research on the Development Strategy of Computational Thinking Ability Under the View of STEM [J]. Modern Educational Technology, 2018.

[16] Regina Enebechi, Nzewi Uchenna. The Effects of Cueing Questions as Instructional Scaffolding of Students' Interest in Biology [J]. 2021, VOL. 1, NO. 288-99. 